\title{
Powering Batteryless Embedded Platforms by Piezoelectric Transducers: A Pilot Study
}

\author{
Michal Prauzek ${ }^{1}$, Jaromir Konecny ${ }^{1}$, Martin Vitasek ${ }^{1}$, Vaclav Bajgar ${ }^{1}$, Petr Musilek ${ }^{2}$ \\ ${ }^{1}$ Faculty of Electrical Engineering and Computer Science, VSB Technical University of Ostrava, \\ Ostrava, Czech Republic \\ ${ }^{2}$ Department of Electrical and Computer Engineering, University of Alberta, \\ Edmonton, AB, Canada \\ michal.prauzek@vsb.cz
}

\begin{abstract}
This contribution presents a pilot study on powering battery-less embedded systems. First, a piezoelectric transducer principle and low-power techniques are reviewed in the background section. In the experimental part, the authors describe a testing setup consisting of piezoelectric transducer, DC/DC converter with energy storage, and evaluation microcontroller platform (FRDM KL25Z). Three types of experiments have been conducted for two voltage configurations including charging speed, continuous operation and discharge test. Results presented in this article concentrate on power supply voltage $(1.8 \mathrm{~V}$ and $3.3 \mathrm{~V})$, total efficiency $(67.16 \%$ and $76.75 \%)$ and operation times (24.28 $\mathrm{s}$ and $15 \mathrm{~s})$ of the embedded system.
\end{abstract}

Index Terms-Energy harvesting; Low-power electronics; Piezoelectric effect; Piezoelectric transducers.

\section{INTRODUCTION}

Powering embedded electronic devices by energy harvesting technologies is an important subject of research due to the widespread implementation of such devices in many application domains [1]. More specifically, many current research challenges stem from the massive deployment of the Internet-of-Things (IoT) sensors (e.g. environmental sensors [1], industrial sensors [4], wearables [2], [3], etc.). This brings the need for development of effective power supply solutions. Battery-less IoT designs offer several significant advantages, including lower price of resulting devices and minimization of their environmental impact (reduced need to use precious metals, avoided disposal of batteries, landfill, and groundwater pollution). The solutions described in this contribution are based on vibration energy harvesting using a piezoelectric converter.

Target application is a battery-less smart IoT sensor that can harvest energy from ambient kinetic energy [5]. Such sensors can be implemented where suitable mechanical energy is available (e.g. transportation vehicles [6], [7],

Manuscript received 18 November, 2018; accepted 16 February, 2019.

This work received funding from the Grant of Student Grant System (VSB-TU Ostrava) reg. no. SP2019/107, "Development of algorithms and systems for control, measurement and safety applications V". It was also supported by the European Regional Development Fund in the Research Centre of Advanced Mechatronic Systems project (No. CZ.02.1.01/0.0/0.0/16_019/0000867) within the Operational Programme Research, Development and Education. industrial facilities [8], etc.). Construction of a battery-less system is a complex task that requires compliance with the energy-neutrality condition [9]. Long-term operational time of a device can be achieved through different technical solutions, including the use of very low-power MCU and low-power wireless communications (LoraWAN, Sigfox, Weightless, etc.).

The proposed solution uses energy harvesting from vibrations using piezoelectric materials. This energy source has energy availability defined by a stochastic model describing the source of kinetic energy and its path to energy converter [10]. The character of harvested energy and its time distribution varies depending on the amount of kinetic energy generated by the source, the length of the path from the source to the converter, and the method of affixing the converter to the source [11]. In this contribution, we are evaluating possibilities to use piezoelectric transducers for powering microcontroller-based embedded systems.

This article is organized into four sections. Section II provides a brief overview of the current state of the art in piezoelectric converters and low-power techniques. Section III describes the experimental setup for evaluating the proposed approach and obtained results. Section IV brings major conclusions and a brief outline of the future work.

\section{BACKGROUND}

\section{A. Piezoelectric Transducer}

Piezoelectric converters are based on the direct piezoelectric effect. Exposing a piezoelectric material to mechanical deformation results in electrical voltage generation, caused by changes in the crystal structure of the material. The amount of obtained energy depends on the type of piezoelectric material and on the size and direction of deformation. A piezoelectric converter often takes the form of an interlocked beam. The beam can be exposed to stress or vibration with a constant or varying frequency [12].

Piezoelectric material (Fig. 1) is usually very rigid and it has high resonance frequencies. Therefore, we use a beam form that transforms a large deviation in the $\mathrm{z}$-axis to smaller deformation $\varepsilon$ in $\mathrm{x}$-axis. A mass is often attached to the tip of a piezo-converter to increase the momentum and thus the amount of harvested energy [13]. 


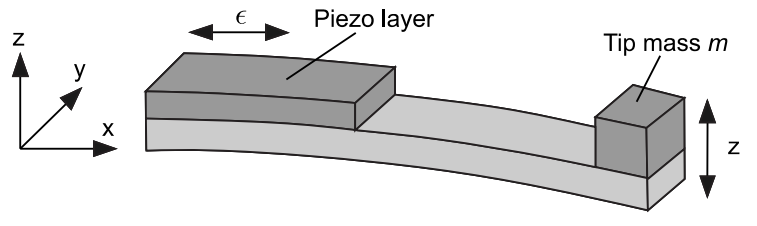

Fig. 1. Piezoelectric transducer construction.

\section{B. Low-Power Methods}

The total energy that a piezoelectric transducer can deliver to embedded systems is relatively small, so the powered system must be designed for a very low-power consumption. Low-power techniques can be divided to several classes according to the levels they operate on [9]:

- Hardware-level techniques;

- Power system techniques;

- Computing system techniques;

- Peripheral system control techniques.

Hardware-level techniques include effective components selection, minimization of static currents, and design of disconnectable peripherals and basic system components. Techniques at the power system level use optimization of maximum power-point tracking, and design of high efficiency DC/DC converters combined with power saving modes. Computing system level techniques include periodic adaptive switching between normal and power saving modes, and energy consumption optimization in normal and power saving modes. Peripheral control system level techniques use adaptive algorithms for sensors duty-cycling, adaptive data storage to non-volatile memories, power switching for individual system components, and eventdriven data transfers. These techniques allow development of efficient low-power devices that can be used in batteryfree designs with limited power available from the environment.

\section{EXPERIMENT AND RESULTS}

\section{A. Experimental Setup Description}

The goal of the experimental work is to evaluate an MCU-driven battery-less embedded system powered by energy harvested using a piezoelectric converter. As described earlier, the piezoelectric transducer is commonly attached to a source of kinetic energy. For testing purposes, a vibration generator was implemented using electrical motor, as shown in Fig. 2.

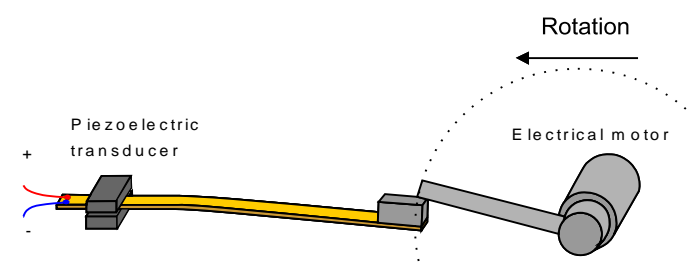

Fig. 2. Kinetic energy excitation by electrical motor hitting piezoelectric beam.

The motor rotates with frequency of $1.33 \mathrm{~Hz}$, emulating energy obtained from the environment. An arm attached to the shaft bends the piezoelectric beam. The beam tip starts vibrating with frequency of $10 \mathrm{~Hz}$ when the arm releases the tip. The resonant frequency of the beam depends on its length and the mass of the weight located at its end. When the resonant frequency of the beam matches the frequency of the kinetic source frequency, the beam works in the optimal regime.

Vibrations of the beam result in dynamic deformations of the piezoelectric material in the inner layer of the piezoelectric converter. Therefore, the kinetic energy is transformed to alternating electrical voltage on transducer output (Fig. 3) based on direct piezoelectric effect.

Figure 4 shows a block diagram of the testing setup. Its input is the kinetic energy harvested by the piezoelectric transducer. Alternating current from the piezoelectric converter enters the energy harvester module.

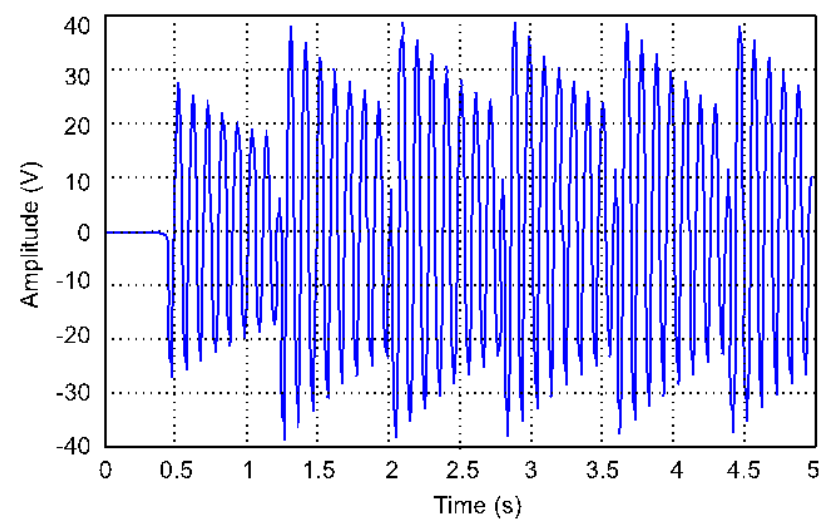

Fig. 3. Output voltage of the piezoelectric transducer excited by the momentum of the electrical motor.

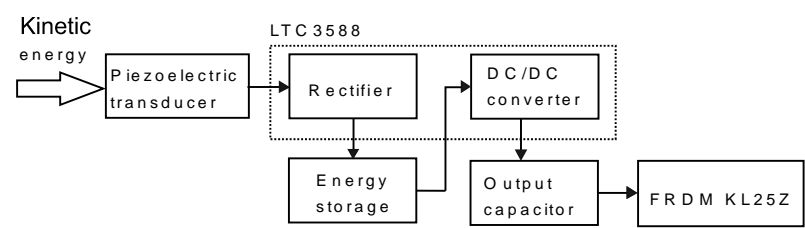

Fig. 4. A block diagram of the testing setup; it describes the path from the kinetic energy transducer to the load represented by the evaluation MCU board (FRDM KL52Z).

The energy conversion and storage module is based on the integrated circuit LTC3588. The output alternating voltage is rectified and then stored to the energy storage device implemented using an electrolytic capacitor with capacitance of $100 \mu \mathrm{F}$. When the energy storage voltage surpasses a preset threshold, the DC/DC converter starts operating in the step-down mode. The DC/DC converter operates with high efficiency of 60-90\%, and it keeps a constant voltage on the output capacitor.

The testing platform, based on the development board FRDM KL25Z with ARM-Cortex M0+ MCU, is powered by the harvested kinetic energy converted by LTC3588, a nanopower energy harvesting power supply. The FRDM module has many features that could draw relatively high power. Therefore, the board was modified and unused peripherals (accelerometer, communication interfaces, etc.) were disconnected to minimize its power consumption. The resulting power supply current drawn by the board is 700800 $\mu \mathrm{A}$ in Very Low-Power Run (VLPR) mode, and $15.3 \mu \mathrm{A}$ in Very Low-Power Stop (VLPS) mode. 
To evaluate the capability of the development board to run programs in VLPR mode, a testing firmware was implemented. A 7th order FIR filter operating in duty-cycle scenario (switching VLPR and VLPS modes in a predefined interval) was implemented as a testing algorithm.

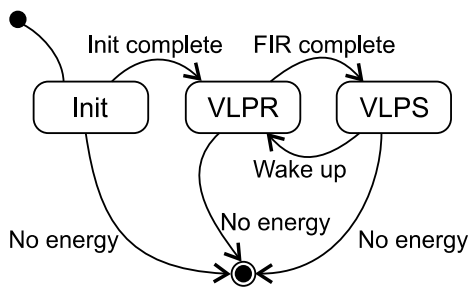

Fig. 5. A finite state machine of the implemented firmware (duty-cycle scenario - switching VLPR and VLPS modes in a predefined interval).

The testing algorithm was implemented as a finite state machine (FSM) (Fig. 5). After the initial kinetic energy conversion, the microcontroller enters the INIT state. After peripheral initialization, the system instantly enters the VLPR mode and runs the FIR filter algorithm. When FIR calculations are completed, the system sets the low-power timer to 4 seconds and the FSM transitions to the VLPS mode, where the system stops all peripherals and awaits a wake-up signal from the low-power timer. This setup allows a 4 seconds duty-cycle operation, when the microcontroller switches between the two low-power modes (VLPR and VLPS). If there is lack of energy for the system operation, it gets a low voltage flag and immediately stops operation.

\section{B. Results and Evaluation}

The following parameters were measured and logged:

- Supply current to development board;

- Time log of energy storage voltage;

- Time log of output capacitor voltage.

High-precision measurement instruments (obtained by DSOX2024A Oscilloscope) were used to capture the very low supply current drawn by the development board. Current measurements were performed using a precise picoamperemeter with analogue voltage output that corresponds to the instantaneous value of the measured current. This output was connected to one of four oscilloscope channels. All voltage time logs were connected to the remaining channels.

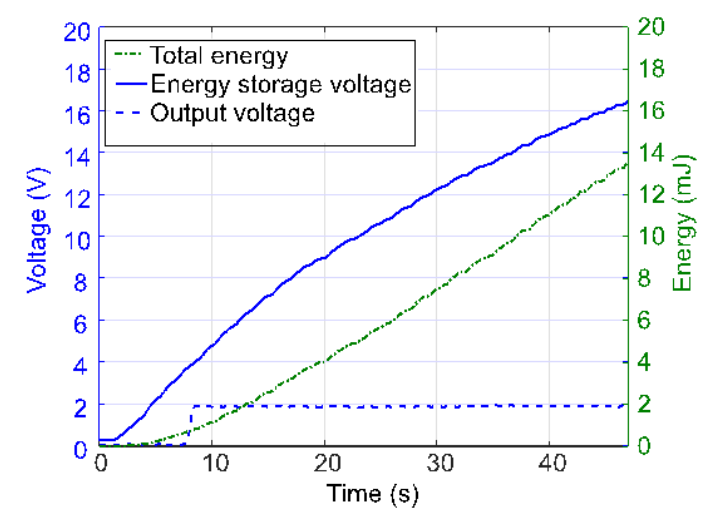

Fig. 6. First experiment - charging the energy storage device without load for $1.8 \mathrm{~V}$ output capacitor.

Supply voltage for KL25Z microcontroller ranges from
1.71-3.6 V. For evaluation purposes, two configurations of the DC/DC converter output voltage $(1.8 \mathrm{~V}$ and $3.3 \mathrm{~V})$ were used to perform three types of experiments. In the following, measurements for the $1.8 \mathrm{~V}$ case are presented in a graphical form. Both configurations are compared in a summary table at the end of this section.

The goal of the first experiment (Fig. 6) was to obtain information about the speed of charging of the energy storage device by the kinetic energy. The electrical motor (Fig. 3) started to rotate when the storage device was completely depleted, and the shaft first hit the transducer arm in 1.25 seconds. The transducer started converting the kinetic energy and the DC/DC converter started operating when the storage device reached a pre-set threshold (in approximately 8 seconds). The energy storage reference voltage of $15 \mathrm{~V}$, corresponding to $11.25 \mathrm{~mJ}$ of energy stored in the capacitor, was reached at time 40.48 seconds, or 39.23 seconds after the first hit of the beam tip.

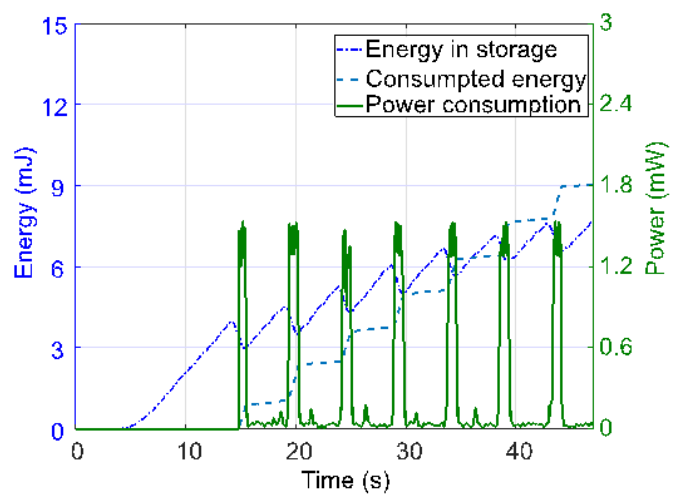

Fig. 7. Second experiment - operation with continuous delivery of kinetic energy for $1.8 \mathrm{~V}$ output capacitor.

The second experiment examined the possibility of permanent system operation with a continuous delivery of kinetic energy. Figure 7 shows the time log of the current stored energy, total energy consumption and instantaneous input power. The piezoelectric converter delivered enough energy for continual operation of the experimental device. It was also observed that when the energy storage voltage raises, the DC/DC converter operates with lower efficiency due to its step-down topology (higher voltage drop causes lower efficiency). Therefore, the delivered energy is in balance with the instantaneous power consumption.

The third experiment demonstrates discharging from the reference state of charge $(11.25 \mathrm{~mJ} / 15 \mathrm{~V})$. After the initialization of the energy buffer, the experimental device was connected, and no energy was supplied from the piezoelectric transducer. The goal was to evaluate the maximum operation time for the experimental setup. Figure 8 shows the details of the instantaneous and total power consumption, as well as the amount of energy remaining in the storage device. In the examined configuration, the system was able to operate for 24.28 seconds.

Overview of the results is presented in Table I for both output capacitor configurations $(1.8 \mathrm{~V}$ and $3.3 \mathrm{~V})$. The numerical results are averages of 10 trial runs for each experiment. For both configurations, the difference between supply currents was not significant. However, due to the different supply voltage, there was a significant difference in 
terms of power consumption: it affects the operation time and lower voltage configuration can prolong the operation by $62 \%$ with same amount of initial energy.

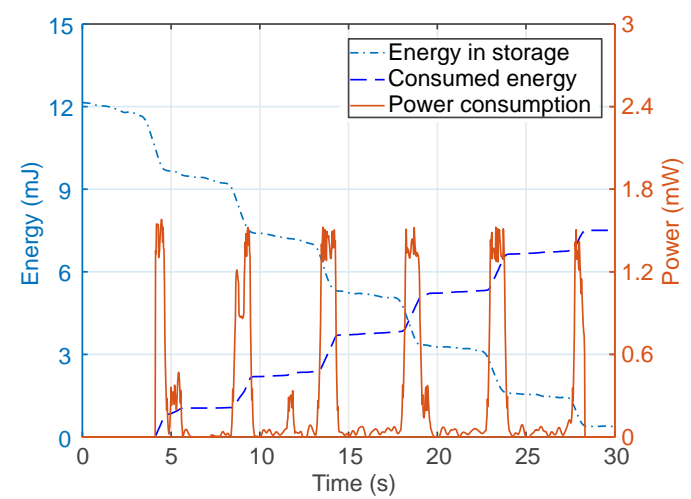

Fig. 8. Third experiment - discharging from reference energy level without delivery of kinetic energy for $1.8 \mathrm{~V}$ output capacitor.

TABLE I. OVERALL OF EXPERIMENTAL TESTING.

\begin{tabular}{|c|c|c|}
\hline & $\mathbf{1 . 8} \mathbf{~ V}$ & $\mathbf{3 . 3} \mathbf{~ V}$ \\
\hline VLPR current & $0.764 \mathrm{~mA}$ & $0.706 \mathrm{~mA}$ \\
\hline VLPS current & $0.018 \mathrm{~mA}$ & $0.020 \mathrm{~mA}$ \\
\hline Average current & $0.167 \mathrm{~mA}$ & $0.164 \mathrm{~mA}$ \\
\hline Average power & $0.298 \mathrm{~mW}$ & $0.539 \mathrm{~mW}$ \\
\hline Operation time & $24.28 \mathrm{~s}$ & $15.00 \mathrm{~s}$ \\
\hline Utilized energy & $7.245 \mathrm{~mJ}$ & $8.089 \mathrm{~mJ}$ \\
\hline Energy loss & $3.538 \mathrm{~mJ}$ & $2.450 \mathrm{~mJ}$ \\
\hline Unused energy & $0.382 \mathrm{~mJ}$ & $0.676 \mathrm{~mJ}$ \\
\hline Total energy & $11.165 \mathrm{~mJ}$ & $11.216 \mathrm{~mJ}$ \\
\hline Total efficiency & $67.16 \%$ & $76.75 \%$ \\
\hline
\end{tabular}

In terms of energy utilization and total energy efficiency, the observation is reverse. The $3.3 \mathrm{~V}$ configuration can utilize more energy than $1.8 \mathrm{~V}$ configuration, i.e. its total energy efficiency is higher. This is caused primarily by the operation efficiency of the DC/DC converter, because the voltage drop is principally lower in the $3.3 \mathrm{~V}$ case.

\section{CONCLUSIONS}

This study explores the possibility to use a piezoelectric energy harvesting transducer for powering MCU-based battery-less embedded systems. The experimental part of this article describes the configuration of a testing system and presents result of three evaluation experiments under two power supply voltage levels $(1.8 \mathrm{~V}$ and $3.3 \mathrm{~V})$. There are two important conclusions based on the observed behaviour of the tested system. First, the $1.8 \mathrm{~V}$ solution has a significantly lower power consumption due to the nature of MCU integrated circuits. When using low voltages to power the MCU, the static consumption is reduced. In addition, the dynamic power consumption can also be lowered using slower clock frequency. Second conclusion, concerning the total rate of utilized energy, is the reverse of the first observation. When using higher output voltage, the DC/DC converter operates with a higher efficiency due to the relatively lower voltage drop from the energy storage device. Therefore, only static consumption is considered, and then higher supply voltage has a substantial advantage in terms of utilized energy and total efficiency. Both observations can be used to select a voltage level appropriate for a given application. In addition, more advanced techniques could be used to further reduce the overall energy consumption of MCU-based battery-less designs, such as dynamic voltage scaling and supercapacitor can be considered as a suitable energy storage element.

\section{REFERENCES}

[1] M. Prauzek, J. Konecny, M. Borova, K. Janosova, J. Hlavica, P. Musilek, "Energy harvesting sources, storage devices and system topologies for environmental wireless sensor networks: A review", Sensors (Switzerland), vol. 18, no. 8, article ID. 2446, 2018. DOI: 10.3390/s18082446.

[2] J. Zhao, Z. You, "Models for 31-mode pvdf energy harvester forwearable applications", Scientific World Journal, vol. 2014, 2014.

[3] K. Suhaimi, R. Ramlan, A. Putra, "A combined softening and hardening mechanism for low frequency human motion energy harvesting application", Advances in Acoustics and Vibration, vol. 2014, 2014. DOI: 10.1155/2014/893496.

[4] X. Zhang, J. Fang, F. Meng, X. Wei, “A novel self-powered wireless sensor node based on energy harvesting for mechanical vibration monitoring", Mathematical Problems in Engineering, vol. 2014, pp. 1-5, 2014. DOI: $10.1155 / 2014 / 642365$.

[5] F. U. Khan and I. Ahmad, "Review of energy harvesters utilizing bridge vibrations", Shock and Vibration, vol. 2016, 2016. DOI: 10.1155/2016/1340402.

[6] D. Maurya, P. Kumar, S. Khaleghian, R. Sriramdas, M. G. Kang, R. A. Kishore, V. Kumar, H. Song, J. J. Park, S. Taheri, S. Priya, "Energy harvesting and strain sensing in smart tire for next generation autonomous vehicles", Applied Energy, vol. 232, pp. 312 322, 2018. DOI: 10.1016/j.apenergy.2018.09.183.

[7] Y. Wang, X. Zhu, T. Zhang, S. Bano, H. Pan, L. Qi, Z. Zhang, Y. Yuan, "A renewable low-frequency acoustic energy harvesting noise barrier for high-speed railways using a helmholtz resonator and a pvdf film", Applied Energy, vol. 230, pp. 52-61, 2018. DOI: 10.1016/j.apenergy.2018.08.080.

[8] K. Kim, J. Y. Cho, D. H. Jeon, J. H. Ahn, S. D. Hong, Y. Jeong, S. Nahm, T. H. Sung, "Enhanced flexible piezoelectric generating performance via high energy composite for wireless sensor network", Energy, vol. 159, pp. 196-202, 2018. DOI: 10.1016/j.energy.2018.06.048.

[9] P. Musilek, M. Prauzek, P. Krömer, J. Rodway, T. Barton̆, "Intelligent energy management for environmental monitoring systems", Smart Sensors Networks: Communication Technologies and Intelligent Applications, pp. 67-94, 2017. DOI: 10.1016/B9780-12-809859-2.00005-X.

[10] X. Li, D. Upadrashta, K. Yu, Y. Yang, "Sandwich piezoelectric energy harvester: Analytical modeling and experimental validation", Energy Conversion and Management, vol. 176, pp. 69-85, 2018. DOI: 10.1016/j.enconman.2018.09.014.

[11] M. Rosa, C. De Marqui Junior, "Modeling and analysis of a piezoelectric energy harvester with varying cross-sectional area", Shock and Vibration, vol. 2014, 2014. DOI: 10.1155/2014/930503.

[12] Y. Liao, J. Liang, "Unified modeling, analysis and comparison of piezoelectric vibration energy harvesters", Mechanical Systems and Signal Processing, vol. 123, pp. 403-425, 2019. DOI: 10.1016/j.ymssp.2019.01.025.

[13] J. H. Ahn, W. S. Hwang, S. Jeong, J. Y. Cho, S. D. Hong, S. J. Hwang, G. J. Song, H. H. Yoo, T. H. Sung, "Nonlinear Piezoelectric Energy Harvester with Ball Tip Mass", Sensors and Actuators, A: Physical, vol. 277, pp. 124-133, 2018. DOI: 10.1016/j.sna.2018.03.015. 